\title{
Revue de littérature des écrits scientifiques portant sur l'improvisation musicale : identification des concepts clés et des recommandations pédagogiques liés à ce domaine A review of musical improvisation scientific literature: Key concepts and pedagogical recommendations
}

\section{Jean-Philippe Després et Francis Dubé}

Volume 15, numéro 1, printemps 2014

L'apprentissage et l'enseignement de la musique : regard francophone international

\section{URI : https://id.erudit.org/iderudit/1033795ar}

DOI : https://doi.org/10.7202/1033795ar

\section{Aller au sommaire du numéro}

\section{Éditeur(s)}

Société québécoise de recherche en musique

\section{ISSN}

1480-1132 (imprimé)

1929-7394 (numérique)

\section{Découvrir la revue}

Citer cet article

Després, J.-P. \& Dubé, F. (2014). Revue de littérature des écrits scientifiques portant sur l'improvisation musicale : identification des concepts clés et des recommandations pédagogiques liés à ce domaine. Les Cahiers de la Société québécoise de recherche en musique, 15(1), 55-66.

https://doi.org/10.7202/1033795ar

\section{Résumé de l'article}

Les recherches théoriques et empiriques sur l'improvisation musicale n'offrent toujours pas de réponse satisfaisante à la question qui préoccupe le plus les praticiens, à savoir : "Comment l'improvisation peut-elle être enseignée de manière efficace ? ». Pour répondre à cette question, nous avons effectué une analyse des écrits scientifiques portant sur l'improvisation musicale. Après avoir appliqué la stratégie proposée par Vallerand et Hess (2000) pour sélectionner les écrits pertinents à notre domaine de recherche, nous avons procédé à une analyse de contenu de ces écrits, en suivant le modèle mixte de L’Écuyer (1987). Notre analyse a permis d'identifier les principales composantes cognitives de l'improvisation musicale et applications pédagogiques proposées dans la littérature pour l'enseignement-apprentissage de cette compétence. De plus, cette recension des écrits nous a permis de constater que certaines composantes cognitives de l'improvisation sont mentionnées par plusieurs chercheurs. Pour cette raison, nous croyons qu'elles devraient être prises en compte lors de l'intégration de l'improvisation musicale à la démarche d'apprentissage de l'élève. Par ailleurs, nous avons également constaté que les fondements empiriques des modèles actuels de l'improvisation ne sont pas suffisamment détaillés, ce qui affaiblit leur validité. Ce constat nous amène à conclure que les effets des applications pédagogiques proposées dans la littérature devraient être vérifiés par la recherche scientifique afin de s'assurer de leur efficacité.
Tous droits réservés @ Société québécoise de recherche en musique, 2014
Ce document est protégé par la loi sur le droit d'auteur. L’utilisation des services d'Érudit (y compris la reproduction) est assujettie à sa politique d'utilisation que vous pouvez consulter en ligne.

https://apropos.erudit.org/fr/usagers/politique-dutilisation/ 


\section{Revue de littérature des écrits scientifiques portant sur l'improvisation musicale : Identification des concepts clés et des recommandations pédagogiques liés à ce domaine}

\author{
Jean-Philippe Després (Université Laval) \\ et Francis Dubé (Université Laval)
}

leur récurrence dans la littérature en improvisation musicale et par le fait qu'il s'agit de deux notions assez différentes pour ne pas être assimilables. Une définition théorique de chacun de ces construits sera présentée ici en vue de démontrer en quoi ils se distinguent et afin de poser les fondements théoriques de notre recherche.

\section{Processus cognitifs - définition}

«La créativité pourrait impliquer davantage que la cognition, mais certains processus cognitifs seraient nécessaires à la quasi-totalité des créativités ${ }^{4}$.»

(Runco 2001, 2892; notre traduction) répondre à la question : «Comment l'improvisation peut-elle être enseignée de manière efficace?». Nous avons réalisé une recension des écrits théoriques et empiriques portant sur le processus de production de l'improvisation musicale dans le but d'identifier les concepts clés liés à ce domaine et les recommandations pédagogiques qui leur sont associées.

\section{Cadre théorique}

Plusieurs termes différents sont proposés dans la littérature pour désigner les composantes cognitives de la créativité. On retrouve, par exemple, les expressions processus (Kozbelt 2011, 474-475), processus réflexifs ${ }^{2}$ (Cropley 2011, 366), processus métacognitifs (Kozbelt 2011, 476), modes de pensée $^{3}$ (Kozbelt 2011,476) ainsi que stratégies et tactiques (Runco 2011, 423-426). La pluralité de ces appellations, qui réfèrent souvent à des phénomènes plus ou moins similaires, entraine une certaine confusion sémantique, d'autant plus que de nombreux auteurs n'explicitent pas clairement le sens qu'ils attribuent à chacune d'elles. Deux composantes fondamentales du processus créatif ont été retenues suite à une analyse préliminaire de la littérature étudiée: les processus cognitifs et les stratégies. Ce choix est justifié par
La cognition réfère aux processus de perception, d'archivage, d'entreposage, d'organisation et d'utilisation de l'information (Martinsen, Kaufmann et Furnham 2011, 214). Plus spécifiquement, Plucker, Runco et Hegarty définissent les processus cognitifs comme étant des «moyens pris par l'esprit humain pour traiter l'information. À un degré relativement simple, ils comprennent l'attention, la perception, la pratique et l'encodage. Les processus plus complexes comprennent les capacités de réflexion d'ordre supérieur telles que la résolution de problèmes ${ }^{5}$ » (Plucker, Runco et Hegarty 2011, 456; notre traduction). Alors que Plucker, Runco et Hegarty (2011) définissent les processus cognitifs comme étant des opérations mentales, les exemples de «processus cognitifs» qu'ils fournissent afin d'illustrer leur définition n'en sont pas à proprement parler. Le terme attention, par exemple, n'est pas une action, ni même un processus, comme pourraient le représenter les expressions être attentif ou se concentrer. Cependant, si l'on retient la première partie de leur définition, les processus cognitifs peuvent se définir comme étant «des actions mentales qui concernent le traitement d'informations».

\footnotetext{
«To emerge from the application of ordinary, fundamental cognitive processes to existing knowledge structures.»

Traduction libre de «thinking processes ».

Traduction libre de «regimes of thought».

«Creative work may involve more than cognition, but certain cognitive processes may be necessary for virtually all creativity.»

«Actions taken by the human mind to process information. At a relatively simple level, includes attention, perception, rehearsal, and encoding. More complex processes include higher-order thinking skills such as problem solving.»
} 
De plus, la distinction qu'ils proposent entre les processus cognitifs simples et les processus cognitifs complexes paraît pertinente. En effet, les processus complexes, tels que la résolution de problème, peuvent être décomposés en une séquence de processus cognitifs simples et, possiblement, de différentes stratégies.

\section{Stratégies - définition}

Les stratégies qui sont utilisées lors du processus créatif exercent une influence directe sur la performance du créateur. Ainsi, l'identification et l'analyse des stratégies utilisées lors du processus créatif permettraient à la fois de mieux comprendre la pensée créative et d'améliorer les interventions visant à développer la créativité (Mumford et Vessey 2011). Selon Lemaire et Fabre, la stratégie peut être définie comme étant «une série de processus utilisés par un individu pour accomplir une tâche cognitive» (2005, 1-2; notre traduction $)^{6}$. Ainsi, alors que les processus cognitifs peuvent être de nature consciente ou non, la stratégie est, par définition, délibérée puisqu'elle est mise en œuvre afin d'atteindre un but précis. Dans le contexte spécifique de l'improvisation musicale, les stratégies permettraient principalement de générer et de sélectionner des idées musicales.

\section{Problématique}

L'intégration de l'improvisation à la formation musicale aurait un effet positif sur différentes facettes du développement de l'apprenant en favorisant l'assimilation des concepts musicaux et la qualité de l'interprétation (Azzara 1992), l'acuité des perceptions auditives (Wilson 1970), l'aisance scénique et la motivation des musiciens (Kenny et Gellrich 2002). Par ailleurs, contrairement à la croyance répandue selon laquelle l'habileté en improvisation musicale résulterait d'aptitudes innées, plusieurs auteurs soutiennent qu'elle peut être améliorée par un entraînement structuré et progressif (Brophy 2001; Kenny et Gellrich 2002; Kratus 1995).

Historiquement, l'improvisation faisait partie intégrante de la l'activité musicale de la Renaissance et des époques baroque, classique et romantique. En revanche, depuis la fin du XIX ${ }^{e}$ siècle (Moore 1992, 61-64) et jusqu'à tout récemment, l'improvisation musicale a pour ainsi dire disparu des salles de concerts vouées à la musique classique $^{7}$. Or, depuis quelques décennies, ce déclin s'est métamorphosé en renaissance. En effet, les efforts conjugués de chercheurs, de musicologues et d'interprètes ont ravivé l'intérêt pour l'improvisation en musique classique (Solis et Nettl 2009, ix-xv, 1-10). Par exemple, certains interprètes - tel le pianiste Robert Levin ${ }^{8}$ (né en 1947), un spécialiste de la période classique qui improvise des ornements et des cadences de concerto en concert - cherchent à reproduire les pratiques d'exécution d'époque, en se fondant sur les avancées de la recherche en performance practice pour intégrer l'improvisation à leurs prestations (Berkowitz 2009, 5-6).

Malgré les nombreux bénéfices de l'improvisation sur le plan pédagogique, les processus cognitifs de ceux qui la pratiquent sont relativement peu documentés (Thompson et Lehmann 2004, 151-154). Or, une meilleure connaissance des processus cognitifs utilisés par les improvisateurs experts permettrait d'élaborer des activités pédagogiques qui favorisent le développement d'un mode de pensée s'apparentant au leur. Ainsi,

[1]'objectif principal de l'enseignement de l'improvisation devrait être de structurer les activités d'apprentissage de manière à ce que les élèves de tous les niveaux puissent expérimenter des façons de penser qui ressemblent à celles des improvisateurs de haut niveau. L'atteinte de cet objectif exige que l'on examine de plus près et que l'on décrive plus clairement non seulement ce que les experts font, mais également comment ils pensent à ce qu'ils font ${ }^{9}$. (Norgaard 2011,124 ; notre traduction)

Quoique peu de recherches empiriques aient été consacrées au processus de génération de l'improvisation en musique classique et que les écrits accessibles aient le plus souvent abordés ce phénomène de façon spéculative (notamment ceux de Clarke 1992; Johnson-Laird 1991, 2002; Kenny et Gellrich 2002; Pressing 1988), des travaux récents ont étayé l'état des connaissances sur l'improvisation dans d'autres contextes musicaux (par exemple, Berkowitz 2009; Chamblee 2008; Mendonça et Wallace 2004; Menezes 2010; Nardone 1996; Norgaard 2008). Ces dernières recherches ont permis d'identifier certains processus cognitifs impliqués lors de l'improvisation musicale, notamment en musique jazz, et de démontrer qu'il s'agit d'un champ de recherche où il y a encore fort à faire.

\footnotetext{
«A strategy is the series of processes an individual uses to accomplish a cognitive task.»

7 Exception faite de la tradition de l'orgue, où l'on peut retracer la pratique ininterrompue de l'improvisation du XIV ${ }^{e}$ siècle jusqu'à aujourd'hui (Bailey 1993).

8 En plus d'être professeur au département de musique de la Harvard University, chercheur et compositeur, Robert Levin mène une carrière internationale en tant que concertiste. Il possède à son actif une discographie de plus de quarante albums.

9 «The primary goal of improvisation instruction should be to structure learning activities in which students at all levels can experience ways of thinking that resemble those of artist-level improvisers. Accomplishing this goal requires that we more closely examine and more clearly describe not only what experts do but also how they think about what they do.»
} 


\section{Corpus étudié}

La présente recension a été circonscrite aux écrits théoriques et empiriques traitant du processus de production de l'improvisation musicale ainsi qu'aux écrits présentant des recommandations pédagogiques pratiques appuyées sur des recherches empiriques. La sélection des écrits les plus pertinents pour notre recherche s'est basée sur la stratégie proposée par Vallerand et Hess $(2000,78)$, avec une adaptation mineure ${ }^{10}$. Cette stratégie adaptée consiste à:

(1) Identifier un écrit récent pertinent à la recherche;

(2) En parcourir la bibliographie afin d'identifier les références pertinentes;

(3) Rechercher, lorsque les bases de données le permettent, les écrits pertinents qui citent cette source;

(4) Reprendre les étapes (2) et (3) pour chacun des écrits retenus ;

(5) Arrêter la recherche lorsque la taille du corpus est satisfaisante ou, si nécessaire, recommencer à l'étape (1) en recherchant un écrit pertinent à la recherche n'ayant pas été relevé lors des étapes précédentes.

Le corpus étudié se divise en trois catégories distinctes: (1) les écrits théoriques; (2) les recherches empiriques sur le processus de production de l'improvisation musicale et (3) les recherches empiriques sur les effets de l'apprentissage de l'improvisation. La présente recension débure par une synthèse de la littérature accessible liée à chacune de ces catégories. Après quoi, les processus cognitifs, les stratégies, ainsi que les recommandations pédagogiques qui ressortent de la littérature théorique et empirique seront exposés.

\section{(1) Écrits théoriques}

Les modèles théoriques ${ }^{11}$ de production de l'improvisation les plus cités dans la littérature sont ceux de Clarke (1988), Johnson-Laird (2002), Kenny et Gellrich (2002) et Pressing (1988). Bien que les principaux résultats présentés ici soient issus de ces quatre modèles, d'autres écrits ont été colligés afin d'obtenir un portrait plus complet du phénomène étudié: les revues de littérature d'Ashley (2009), Azzara (2002), Gabrielsson (2003), Lehmann, Sloboda et Woody
(2007), ainsi que les articles de Barrett (1998), Brophy (2001), Covington (1997), Johnson-Laird (1991), Kratus (1991, 1995), Monk (2012), Moore (1992), Pressing (1984, 1998), Sarath (1996), Sawyer (2000, 2011) et Scott (2007).

\section{(2) Recherches empiriques - processus de production de l'improvisation musicale}

Si les écrits théoriques abordent souvent l'improvisation dans une perspective plutôt générale, les recherches empiriques se concentrent, quant à elles, sur un objet d'étude circonscrit par une ou plusieurs des variables suivantes: le style musical (par exemple, jazz ou classique), le degré d'expérience (débutant ou avancé) ou la tâche d'improvisation à réaliser (en imposant un certain nombre de contraintes, comme une grille harmonique préétablie ou une indication de tempo). La diversité des méthodes employées dans le cadre de ces recherches n'a d'égale que celle des sujets traités.

Les recherches accessibles portant sur l'expertise dans le domaine de l'improvisation musicale classique tonale, notre objet d'étude, sont peu nombreuses: nous n'en avons repéré que trois (Bailey 1993; Berkowitz 2009; Chamblee 2008). Afin de permettre l'analyse d'un corpus substantiel, cette recension couvre donc les écrits relatifs à la musique occidentale dans son ensemble, en incluant les recherches portant sur l'improvisation jazz (Berliner 1994; Csikszentmihalyi et Rich 1997; Friberg et Sundström 2002; Hargreaves, Cork et Setton 1991; Limb et Braun 2008; Mendonça et Wallace 2004; Nardone 1996; Norgaard 2008, 2011), l'improvisation libre (Menezes 2010) et sans restriction stylistique (Biasutti et Frezza 2009). Cette recension intègre également les recherches dans le cadre desquelles les participants ont réalisé une tâche improvisée artificielle, ne répondant pas aux exigences esthétiques d'un style musical réel ${ }^{12}$.

Le processus de production de l'improvisation musicale a été appréhendé sous deux perspectives épistémologiques principales: (1) les techniques d'imagerie médicale, telles que l'imagerie par résonnance magnétique fonctionnelle $\left(\operatorname{IRMf}^{13}\right)$, et (2) les méthodes issues du domaine de la recherche qualitative. Dans ce dernier cas, la méthode de collecte de données la plus fréquemment employée est le protocole verbal rétrospectif ${ }^{14}$.

\footnotetext{
10 Notre adaptation comprend deux modifications par rapport à la stratégie originale. D'une part, les étapes 3 et 4 sont interverties afin de favoriser l'accès aux écrits récents et à leurs références. D'autre part, nous avons ajouté l'étape 5 dans le but d'inclure également les écrits pertinents qui ont été relativement peu cités dans la littérature.

11 Les modèles théoriques sont des écrits de nature spéculative qui illustrent certains aspects du processus de production de l'improvisation musicale.

12 En utilisant ce type de protocole, les chercheurs peuvent, par exemple, chercher à identifier les zones cérébrales qui agissent lors de l'idéation de la musique improvisée (Berkowitz et Ansari 2008, 2010).

13 L'IRMf a notamment été utilisée dans les recherches de Berkowitz et Ansari (2008, 2010) et de Limb et Braun (2008).

14 Le protocole verbal consiste en l'explicitation, par un individu, des processus mentaux auxquels il fait appel lors de l'exécution d'une tâche donnée. On retrouve deux types de protocoles verbaux : (1) les protocoles simultanés, où le participant décrit à voix haute ses processus mentaux alors même qu'il y fait appel, et (2) les protocoles rétrospectifs, qui sont récoltés après que la tâche soit terminée, généralement dans un laps de temps se voulant le plus court possible (Lemaire et Fabre 2005). Dans le domaine de l'improvisation musicale, le protocole verbal rétrospectif a été employé, entre autres, par Mendonça et Wallace (2004) et Norgaard (2008).
} 


\section{(3) Recherches empiriques - effets de l'apprentissage de l'improvisation}

Certains écrits portant sur les effets de l'improvisation musicale dans un contexte pédagogique ont été retenus (Azzara 1992; Dos Santos et Del Ben 2004; Guilbault 2009; Koutsoupidou et Hargreaves 2009; Mason 2005; McPherson 1993; Montano 1983; Rowlyk 2008; Whitman 2001). Le plus souvent, ces recherches s'appuient sur un devis quantitatif de type «pré-test/post-test ${ }^{15}{ }^{15}$ afin de vérifier les effets d'un traitement donné (par exemple, une formation en improvisation) sur d'autres variables (par exemple, l'acuité des perceptions auditives ou la motivation de l'apprenant).

\section{Analyse des données}

Nous avons procédé à une analyse de contenu du corpus, en suivant le modèle mixte de L'Écuyer $(1987,59)$. Les six grandes étapes de l'analyse de contenu selon L'Écuyer sont les suivantes:

(1) Lecture préliminaire et établissement d'une liste des énoncés;

(2) Choix et identification des unités de classification;

(3) Processus de catégorisation et de classification;

(4) Quantification et traitement statistique;

(5) Description scientifique;

(6) Interprétation des résultats.

(L'Écuyer 1987, 54-55)

$\mathrm{Vu}$ le nombre relativement faible de catégories et de sous-catégories répertoriées, l'étape 4 de L'Écuyer a été exclue du processus d'analyse. L'analyse des données a permis de dégager cinq processus cognitifs, neuf stratégies d'improvisation, ainsi que onze recommandations pédagogiques pour l'enseignement de l'improvisation.

\section{Résultats}

\section{Processus cognitifs mis en auvre lors de l'improvisation}

Cinq processus cognitifs pourraient être mis en œuvre par les musiciens experts lors de l'improvisation:

(1) Anticipation;

(2) Exécution;
(3) Monitoring ${ }^{16}$;

(4) Rappel;

(5) Évaluation.

Bien que l'improvisation se déroule en temps réel (Ashley 2009,413-416; Johnson-Laird 2002, 415; Kenny et Gellrich 2002, 117-120; Pressing 1988, 136) et qu'elle demande au musicien à la fois de générer, d'exécuter et d'évaluer des idées musicales (Johnson-Laird 2002, 422), il semble que les différents processus cognitifs identifiés dans la littérature soient mis en œuvre à tour de rôle et non pas simultanément (Kenny et Gellrich 2002, 123-124; Monk 2012, 89).

Selon Kratus (1995, 30-31), la capacité qu'a le musicien improvisateur d'anticiper la conséquence sonore de ses gestes distingue sa pratique de la simple exploration sonore. L'anticipation est le processus cognitif au cours duquel le musicien planifie certaines caractéristiques de son improvisation, comme la densité des notes, le registre ou la structure harmonique (Kenny et Gellrich 2002, 124; Norgaard 2008, 62). Le processus d'anticipation peut s'accomplir à différentes échelles. En effet, l'anticipation peut concerner les prochaines notes à jouer (court terme), la prochaine phrase ou période musicale (moyen terme) ou encore l'improvisation dans son ensemble, du moment présent jusqu'à la fin de la performance (long terme) (Kenny et Gellrich 2002, 124). Par ailleurs, le processus d'anticipation peut être divisé en deux phases: (1) génération des idées et (2) sélections des idées (Johnson-Laird 2002, 421). Lors de la génération des idées, le musicien élabore une ou plusieurs idées musicales à l'aide de ses connaissances stylistiques. Par la suite, lors de la sélection des idées, il choisit une idée parmi celles qu'il aura générées. Selon Johnson-Laird (2002, 421), pour que l'improvisation puisse se dérouler en temps réel, cette sélection devrait se faire de façon aléatoire et involontaire afin de ne pas trop solliciter la mémoire du musicien.

Après avoir établi un plan, le musicien doit le mettre à exécution. L'exécution est le processus cognitif qui permet aux idées musicales de se manifester en sons (Thompson et Lehmann 2004, 151-152). Au cours de ce processus, une série de signaux est envoyée du cerveau au corps afin de réaliser le plan qui vient d'être élaboré. La capacité de transformer les idées musicales en sons est l'une des habiletés qui seraient maitrisées par l'improvisateur expert (Kratus 1995, 29).

15 Dans un devis «pré-test/post-test», un échantillon de la population est soumis à une variable indépendante ou traitement (par exemple, une formation en improvisation). Les variables dépendantes (comme la motivation de l'apprenant) sont mesurées avant et après l'exposition au traitement (Vallerand et Hess 2000).

16 Nous avons choisi de retenir le terme anglais monitoring, car il a un sens plus général que sa traduction française recommandée «monitorage», dont l'usage est davantage restreint aux domaines médical et technologique. 
Alors qu'il s'exécute, le musicien peut faire la «surveillance» de son improvisation, c'est-à-dire concentrer son attention sur la conséquence sonore de ses gestes (Norgaard 2008, 65-69). Dans la littérature, ce processus est appelé monitoring. Une des conditions essentielles pour faire appel au monitoring est de posséder l'aisance nécessaire pour passer rapidement d'un processus à un autre (Monk 2012, 93).

Le rappel est le processus enclenché lorsque le musicien se remémore des éléments joués précédemment au cours de son improvisation (Kenny et Gellrich 2002, 124). Tout comme l'anticipation, le rappel peut concerner les notes précédentes (court terme), la phrase ou la période précédente (moyen terme) ou encore l'ensemble de l'improvisation, du début jusqu'au moment présent (long terme) (Kenny et Gellrich 2002, 124).

Le processus d'évaluation est mis en œuvre en parallèle avec d'autres processus. Lors de l'anticipation, le musicien peut faire appel à l'évaluation afin de juger de la qualité des idées qu'il aura générées. Lors du monitoring, l'évaluation lui permet de vérifier, d'une part, l'adéquation entre le plan qu'il avait préalablement élaboré et les conséquences sonores de ses gestes (Norgaard 2008, 178) et, d'autre part, la pertinence de la réalisation concrète de ses idées. Lors du rappel, l'évaluation des idées qui ont été jouées précédemment permet au musicien de décider s'il s'inspirera, ou non, de ces dernières afin d'anticiper la suite de son improvisation.

\section{Stratégies mises en auvre lors de l'improvisation}

Il se dégage des écrits consultés neuf stratégies pouvant être utilisées par les musiciens experts lorsqu'ils produisent une improvisation. Après avoir été énumérées, ces neuf stratégies seront définies tour à tour:

(1) Planification générale;

(2) Utilisation de la banque d'idées;

(3) Recombinaison;

(4) Improvisation hiérarchique;

(5) Feedback;

(6) Génération interrompue ;

(7) Utilisation du potentiel créatif des erreurs;

(8) Priorité mélodique;

(9) Priorité harmonique.
Alors que l'attention du musicien qui débute son apprentissage en improvisation est principalement concentrée sur de courts segments, celle de l'expert est plus globale, ce qui lui permet de concevoir une planification générale de l'improvisation avant de commencer à jouer (Hargreaves, Cork et Setton 1991, 52-53; Norgaard 2008, 62-65). Cette stratégie de planification générale lui permet de déterminer la structure globale de son improvisation, de même que certaines caractéristiques des phrases, des contours mélodiques ainsi que des résolutions harmoniques et rythmiques à venir (Barrett 1998, 607).

L'automatisation de sous-routines d'improvisation, ou banque d'idées, permet de libérer l'attention nécessaire à la planification d'une stratégie globale de l'œuvre (Hargreaves, Cork et Setton 1991, 63). Une des stratégies mentionnées de façon récurrente dans la littérature spécialisée est l'utilisation de la banque d'idées ${ }^{17}$. En effet, l'apprentissage de l'improvisation - que ce soit dans la musique classique persane (Nettl 2009), dans les œuvres de la Première école viennoise (Berkowitz 2009) ou en musique jazz ${ }^{18}$ (Norgaard 2008) - passe généralement par la mémorisation d'un répertoire de motifs. Il s'agit d'idées musicales automatisées qui peuvent être utilisées en temps réel lors de l'improvisation musicale (Ashley 2009, 415). Par exemple, les ornements fréquemment utilisés par les musiciens de l'époque baroque (Bailey 1993, 19-21) faisaient partie de leur banque d'idées. Ainsi, la banque d'idées est composée de formules entreposées en mémoire à long terme qui peuvent soit être répétées de façon intégrale par le musicien (Kenny et Gellrich 2002, 117-126) ou encore adaptées sur le vif ${ }^{19}$ lors de l'improvisation (Norgaard 2008, 69-73).

En plus d'utiliser intégralement les motifs contenus dans sa banque d'idées, le musicien peut également allier les caractéristiques de différents motifs en temps réel lors de l'improvisation. Cette dernière stratégie est appelée recombinaison (Berkowitz 2009, 82-97).

Par ailleurs, le musicien peut aussi s'inspirer des structures harmoniques et mélodiques de la pièce à partir de laquelle il improvise comme matériau créatif. Cette stratégie est nommée improvisation hiérarchique par Clarke (1988, 8-9). La variation est un exemple typique d'improvisation hiérarchique (Kenny et Gellrich 2002, 122).

Le feedback ${ }^{20}$ (Kenny et Gellrich 2002, 124) fait également partie des stratégies pouvant être utilisées lors de l'improvisation. Le musicien y fait appel lorsqu'il s'inspire des caractéristiques des idées musicales qu'il a créées précédemment pour développer de nouvelles idées.

17 Cette stratégie est également dénommée sélection du répertoire par Clarke (1988).

18 En jazz, les motifs contenus dans la banque d'idées sont communément dénommés licks.

19 Cette adaptation se ferait généralement à l'aide des principes de l'organisation tonale (Ashley 2009).

20 Cette stratégie est également appelée stratégie associative par Clarke (1988). 
Par exemple, lorsque le musicien répète un motif original en séquences transposées, il fait appel au feedback (Kenny et Gellrich 2002, 124). L'utilisation de cette stratégie implique par conséquent la mise en œuvre des processus cognitifs du rappel et de l'anticipation (Kenny et Gellrich $2002,124)$. D'autre part, le modèle complexe de production de l'improvisation que propose Pressing (1988) est basé principalement sur l'utilisation de la stratégie associative.

La littérature théorique fait également état de la stratégie qui consiste à élaborer du matériel contrastant par rapport à ce qui vient d'être joué, ce que Pressing $(1988,157)$ nomme la génération interrompue ${ }^{21}$. Lors du feedback, les nouvelles idées étaient développées dans la continuité de ce qui avait été joué auparavant, tandis que lors de la génération interrompue, elles sont élaborées de manière à créer une rupture dans le discours musical et d'éviter ou de rompre la monotonie.

Csikszentmihalyi et Rich (1997) ont analysé trois entretiens menés avec des improvisateurs experts de différents horizons : Ravi Shankar ${ }^{22}$, Oscar Peterson ${ }^{23}$ et Gunther Schuller ${ }^{24}$. Dans le cadre de ces entretiens, le pianiste jazz Oscar Peterson évoque une stratégie qu'il juge importante pour tout musicien improvisateur: savoir utiliser le potentiel créatif des erreurs. Lorsqu'il fait appel à cette stratégie, le musicien exploite les évènements inattendus comme des agents créatifs. À titre d'exemple, il pourrait utiliser une note a priori erronée comme pivot vers une modulation qui n'était pas prévue au départ.

La priorité mélodique est la stratégie que le musicien utilise lorsqu'il se base sur le contour mélodique pour déterminer la hauteur des notes durant l'improvisation (Norgaard 2008, 80-81). En d'autres mots, lorsqu'il fait appel à cette stratégie, le musicien adopte une approche qui est davantage horizontale, le plus souvent en faisant appel à des gammes.

Enfin, la stratégie de la priorité harmonique est une approche verticale de l'improvisation. En effet, lorsqu'il fait appel à cette stratégie, le musicien se base sur les notes structurelles des accords ${ }^{25}$ pour déterminer les hauteurs des notes lors de l'improvisation (Norgaard 2008, 76-79). Les interviews réalisées par Norgaard (2008) ont montré que la stratégie de la priorité harmonique est d'une importance capitale pour les improvisateurs jazz: ils l'utilisent plus fréquemment que la stratégie de la priorité mélodique.

\section{Recommandations pédagogiques}

La figure 1 illustre la relation entre les stratégies utilisées par les improvisateurs experts, les recommandations pédagogiques qui permettraient à l'apprenant de s'approprier ces stratégies et les processus cognitifs qui sont principalement impliqués lors de la mise en œuvre de ces stratégies. De plus, la boucle formée par les processus cognitifs dans la section située à droite de la figure démontre la circularité qui opère entre les différentes composantes du processus de production de l'improvisation musicale. En effet, lors de l'improvisation, ce qui a été joué précédemment détermine en grande partie ce qui sera joué ultérieurement.

\section{Application des stratégies}

Si l'enseignant souhaite développer la capacité d'établissement d'un plan d'ensemble de l'improvisation projetée, il aura avantage à créer un contexte d'improvisation qui soit confortable pour l'apprenant. En d'autres mots, ce dernier devra d'abord maîtriser parfaitement les contraintes harmoniques, rythmiques et stylistiques de l'improvisation projetée afin de pouvoir se concentrer sur la planification globale de son improvisation avant même de commencer à jouer (Norgaard 2008, 193).

Pour développer la banque d'idées de l'apprenant, le professeur cherchera à augmenter la taille et la flexibilité de sa collection de connaissances. Pour ce faire, il pourra suggérer à l'élève de mémoriser et d'apprendre à transposer, à l'oreille, un répertoire de lignes de basses, de mélodies et de progressions harmoniques (Kenny et Gellrich 2002, 129132).

Afin de recourir aux stratégies de recombinaison, d'improvisation hiérarchique, de feedback et de génération interrompue, l'improvisateur doit être en mesure d'analyser rapidement les caractéristiques fondamentales de différents segments musicaux et de pouvoir les assimiler en temps réel. Pour améliorer la capacité de l'apprenant à faire appel à ces stratégies, l'enseignant créera deux types d'activités complémentaires $^{26}$ : (1) l'étude d'un concept fondamental et (2) la création d'un cadre de contraintes minimales et implicites.

En fait, l'étude d'un concept fondamental (CF) implique l'identification préalable par l'enseignant d'un CF adapté au niveau de l'apprenant (par exemple, un ornement, les

\footnotetext{
21 Traduction libre d' «interrupt generation ».

22 Ravi Shankar (1920-2012) est un sitariste indien qui, en plus d'avoir contribué au développement de la technique de son instrument, a mené une carrière internationale. Il a été le musicien indien le plus populaire de son époque (Slawek s.d.).

23 Oscar Peterson (1925-2007) est un pianiste de jazz canadien reconnu pour sa technique exceptionnelle (Dobbins s.d.).

24 Gunther Schuller (né en 1925) est un compositeur et chef d'orchestre qui a inventé l'expression third stream pour désigner la musique qui combine des éléments de jazz et de musique classique (Carnovale et Dyer s.d.).

25 Traduction libre de «guide tones».

${ }^{26}$ Ces activités peuvent être travaillées isolément ou de façon concomitante.
} 
Figure 1: Identification des composantes cognitives et pédagogiques liées à l'improvisation musicale.

\section{Stratégies \\ Planification générale \\ (Norgaard 2008 \\ Recommandations pédagogiques \\ Dans un contexte maitrîsé \\ - établir un plan global de l'improvisation \\ (Norgaard 2008)}

\section{Utilisation de la banque d'idées \\ (Norgaard 2008)}

\section{Mémorisation et transposition}

- de progressions harmoniques

- de mélodies
(Thompson et Lehmann 2004)

\begin{tabular}{|l|l}
$\begin{array}{l}\text { Recombinaison } \\
\text { (Berkowitz 2009) }\end{array}$ \\
\hline
\end{tabular}

\section{Improvisation hiérarchique}

(Clarke 1988)

\section{Feedback \\ (Kenny et Gellrich 2002)}

\section{Génération interrompue \\ (Pressing 1988)}

\section{Utilisation du potentiel créatif des erreurs}

(Csikszentmihalyi et Rich 1997)

Priorité mélodique

(Norgaard 2008)

\section{Priorité harmonique} (Norgaard 2008

\section{Concept fondamental (CF)}

- mémoriser des motifs comportant le CF

- créer des motifs à l'aide du CF

(Norgaard 2008)

\section{Cadre de contraintes minimales et implicites}

Improviser :

- des articulations

- en s'inspirant des motifs d'une autre mélodie

- un contre-chant

- à l'intérieur d'un registre donné

- etc.

(Barrett 1998; Dos Santos et Del Ben 2004)

\section{Cohérence rétrospective}

- rendre cohérentes les idées a posteriori, par exemple, en répétant une note erronée (Gioia 1988)

\section{Cadre déstabilisant}

- difficulté progressive

- proposer des avenues d'action alternatives

(Barrett 1998)

\section{Sur une pédale harmonique}

- explorer les sonorités de diverses gammes

(Dos Santos et Del Ben 2004)

\section{Sur une progression harmonique}

- improviser seulement avec des notes structurelles

- puis ajouter des notes ornementales

(Norgaard 2008)

\section{Plan d'ensemble \\ - élaboré a priori \\ (Norgaard 2008)}

Improvisation avec les autres

- avec un mentor (Young 2003)

- avec accompagnement (Guilbault 2009)

- en alternance accompagnateur/soliste (Barrett 1998)

\section{Évaluation de l'improvisation}

- en fonction du plan global élaboré a priori

(Norgaard 2008)

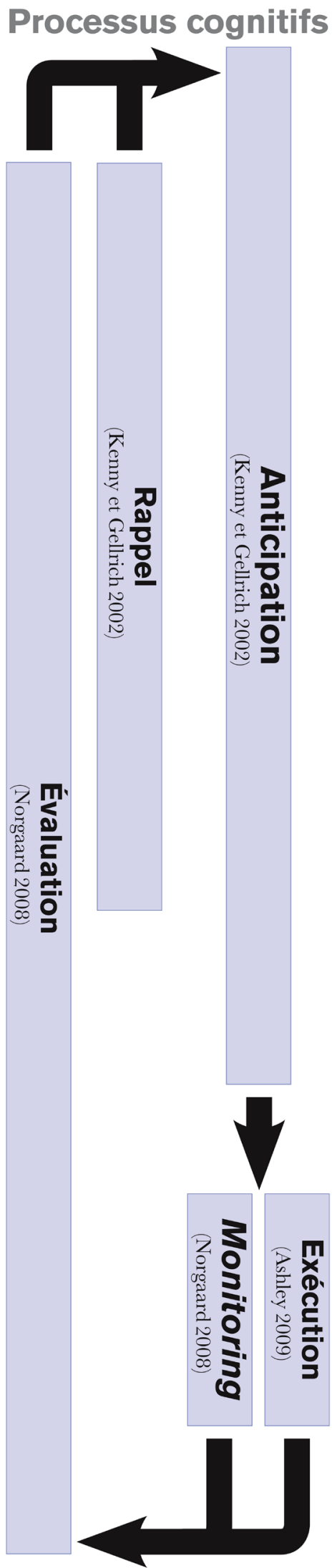


notes structurelles (guide tones), une formule arpégée, les upper structures, etc.). Ensuite, le pédagogue devra amener l'élève à mémoriser plusieurs motifs comportant le CF en apprentissage et à créer lui-même de nouveaux motifs à partir de ce même concept (Norgaard 2008, 191-193 ; 2011, 123-124). Une fois cette étape maîtrisée, il pourra inventer des motifs comportant le CF étudié en temps réel, au cours de l'improvisation.

La création d'un cadre de contraintes minimales et implicites exige également de l'enseignant l'établissement de règles claires régissant l'improvisation. Lorsqu'elles sont bien dosées, les contraintes favorisent la créativité du musicien, en lui permettant de transcender ses habitudes. En effet, si l'enseignant ne propose aucune contrainte à l'élève (par exemple en limitant ses instructions à: «Maintenant,improvise quelque chose»), l'apprenant risque d'expérimenter une angoisse de la page blanche musicale, dans un contexte insécurisant, ce qui pourrait miner sa confiance en soi. Inversement, un cadre de contraintes trop serré risque d'entraver sa créativité. Lorsque l'enseignant propose des activités d'improvisation, il doit donc établir un certain nombre de contraintes et veiller à ce que cellesci soient assimilées par le ou les apprenant(s), de façon à ce qu'elles n'aient pas à être négociées lors du processus créatif. Par exemple, l'enseignant pourrait demander à l'apprenant ou aux apprenants de ne jouer que sur les notes noires du clavier, ou encore "d'improviser des articulations, des variations mélodiques ou rythmiques sur une mélodie familière, une mélodie à partir des motifs caractéristiques d'une autre pièce, un contre-chant ou un accompagnement harmonique sur une mélodie donnée, une mélodie à l'aide d'outils prédéterminés (gamme, arpège, etc.).» (Dos Santos et Del Ben 2004, 268).

Pour encourager l'utilisation du potentiel créatif des erreurs chez son élève, l'enseignant doit veiller (1) à développer chez celui-ci la cohérence rétrospective (Gioia 1990, 61) et (2) à le faire improviser à l'intérieur d'un cadre déstabilisant.

En effet, lors de l'improvisation musicale, la cohérence des idées peut être assurée après qu'elles aient été jouées. Il s'agit du concept que Gioia $(1990,61)$ nomme la cohérence rétrospective. À titre d'exemple, en répétant une note $a$ priori erronée, l'instrumentiste laisse croire à l'auditeur qu'elle était intentionnelle. Pour entraîner l'apprenant à faire appel à cette stratégie, il est suggéré de l'inviter à simuler une fausse note puis de tenter ensuite de la justifier musicalement. Pour ce faire, il pourra par exemple la répéter dans la phrase suivante ou encore créer une séquence à l'aide d'un motif comprenant la fausse note en question, en la résolvant ensuite. Ainsi, en développant sa capacité d'utilisation du potentiel créatif de ses erreurs, l'apprenant n'a plus à craindre les aléas de ses choix, car il sait dorénavant qu'il est en mesure de faire des essais téméraires et de s'ajuster aux situations imprévues (Barrett 1998, 610-611).

Par ailleurs, l'enseignant peut créer un cadre musical déstabilisant afin d'éloigner l'apprenant de ses habitudes et de l'inciter à prendre des risques. Ce faisant, il devrait veiller à (1) présenter les éléments déstabilisants de manière progressive afin qu'ils représentent un défi surmontable, ainsi qu'à (2) proposer des avenues d'actions alternatives (Barrett 1998, 607-610). Par exemple, si l'enseignant choisit de changer au dernier moment la tonalité de la pièce afin de déstabiliser l'apprenant, il optera pour une tonalité accessible à ce dernier. Par ailleurs, il aura préalablement doté l'apprenant d'outils lui permettant de transposer aisément ses idées musicales ou d'en improviser de nouvelles dans la tonalité choisie. Proposer des défis réalistes est un aspect important puisque l'élève qui expérimente une réussite lors d'une improvisation aura tendance à continuer à être musicalement créatif (Rosenbrock 2002, cité par Lehmann, Sloboda et Woody 2007, 141) et à oser prendre des risques. Il développera ainsi son sentiment d'auto-efficacité (Bandura 1997).

L'enseignement de la stratégie de la priorité mélodique se fera en invitant l'apprenant à improviser sur une pédale harmonique et à explorer les différentes gammes pouvant être utilisées sur cet accord, tout en se concentrant sur le contour mélodique de l'improvisation.

Enfin, le professeur d'instrument devra également veiller à faire appliquer la stratégie de la priorité harmonique à son élève puisqu'il s'agit d'une des stratégies les plus importantes pour les improvisateurs jazz (Norgaard 2008, $76-79,184)$. Pour ce faire, l'activité pédagogique se fera à partir d'une progression harmonique donnée. Dans un premier temps, il invitera l'apprenant à ne jouer que les notes structurelles des accords, car elles constitueront la charpente de son improvisation. Ensuite, lorsque cette habileté sera maîtrisée, il pourra lui proposer de s'exercer à relier ces notes structurelles entre elles à l'aide de notes ornementales.

\section{Intégration des processus cognitifs}

Le processus cognitif de monitoring peut être amélioré en exerçant l'élève à respecter le plan global de l'improvisation qu'il aura élaboré a priori (Norgaard 2008, 193). Pour ce faire, ce dernier devra prévoir, avant de commencer à jouer, les grandes lignes de son improvisation et chercher à respecter, tout au long de son improvisation, le plan qu'il aura préalablement élaboré et noté.

Une des approches efficaces pour progresser en improvisation est d'improviser avec les autres. Cette approche permet d'améliorer la capacité de monitoring de l'élève, qui exige qu'il soit capable de passer rapidement d'un processus 
cognitif à un autre. De fait, lorsque l'apprenant improvise en compagnie de son enseignant ou d'un mentor, il intègre certaines caractéristiques du jeu de ce dernier (Young 2003, 184-186). C'est pourquoi l'enseignant devrait prendre le temps de jouer et d'improviser avec son élève, et non seulement de lui donner des consignes et de la rétroaction sur ses improvisations. De plus, autant que possible, le professeur devrait proposer à l'élève de s'exercer à improviser à l'aide d'un accompagnement harmonique, ce qui aurait un effet significatif et positif sur le développement de l'habileté à improviser (Guilbault 2009, 86-89). Ces deux types d'apprentissage peuvent facilement se combiner si l'enseignant joue lui-même l'accompagnement sousjacent au lieu d'utiliser un enregistrement ou un séquenceur. Dans le même ordre d'idée, l'enseignant et l'apprenant peuvent alterner les rôles d'accompagnateur et de soliste, en partageant leurs impressions à voix haute afin que l'apprenant puisse mieux saisir ce que chacun attend de l'autre (Barrett 1998, 617).

Finalement, le pédagogue pourra améliorer la capacité d'évaluation de l'élève en lui demandant d'estimer dans quelle mesure il a respecté le plan global élaboré au départ (Norgaard 2011, 123-124). Advenant le cas où il n'aurait pas respecté intégralement son plan, l'apprenant pourra également évaluer si ses digressions constituaient des améliorations, ou non, par rapport au plan original.

\section{Conclusion}

La plupart des processus cognitifs et des stratégies identifiés dans la littérature sont récurrents d'un auteur à l'autre, en dépit du fait que des appellations différentes soient souvent utilisées pour dénommer un même concept. Au-delà de ces différences sémantiques, ces composantes cognitives devraient être prises en compte dans une pédagogie de l'improvisation musicale, puisqu'étudier les processus cognitifs utilisés par les experts dans un domaine donné permet d'élaborer des activités pédagogiques qui favorisent le développement d'un mode de pensée s'apparentant au leur (Norgaard 2011, 124).

Par ailleurs, il est difficile de déterminer dans quelle mesure les modèles théoriques de l'improvisation proposés à ce jour dans la littérature reposent sur des données empiriques, puisque les auteurs ne font pas toujours état des fondements scientifiques sur lesquels s'appuient leurs modèles. Les processus cognitifs ou les stratégies dont ils font mention trouvent toutefois le plus souvent écho dans la littérature empirique ou encore dans d'autres modèles théoriques. Par exemple, la cohérence rétrospective de Gioia (1990) et l'utilisation des erreurs à des fins créatives
(Csikszentmihalyi et Rich 1997) sont deux stratégies assez semblables et la stratégie de feedback (Kenny et Gellrich 2002) est également discutée par Clarke (1988), qui la nomme plutôt génération associative ${ }^{27}$.

Il demeure que les effets des applications pédagogiques proposées dans la littérature devraient être validés par la recherche scientifique, afin d'en mesurer l'efficacité. De plus, les protocoles des recherches qui ont mesurés scientifiquement l'effet de l'improvisation sur d'autres variables (notamment Azzara 1992; Dos Santos et Del Ben 2004; Koutsoupidou et Hargreaves 2009; Rowlyk 2008; Wilson 1970) devraient être appliqués dans d'autres contextes (par exemple, à d'autres instruments ou à d'autres niveaux d'apprentissage) afin de vérifier dans quelle mesure leurs résultats sont généralisables. Finalement, les relations entre les stratégies, les recommandations pédagogiques et les processus cognitifs qui ont été proposés dans le présent article devraient également être vérifiées par des recherches empiriques. Il serait particulièrement important de mesurer l'effet des diverses approches pédagogiques présentées sur la capacité de l'apprenant à mettre en œuvre les différentes stratégies que nous avons relevées. Il reste donc beaucoup à faire dans ce domaine.

\section{RÉFÉRENCES}

Ashley, Richard (2009). «Musical Improvisation», dans Susan Hallam, Ian Cross et Michael Thaut (dir.), The Oxford Handbook of Music Psychology, New York, Oxford University Press, p. 413-420.

Azzara, Christopher D. (1992). «The Effect of AudiationBased Improvisation Techniques on the Music Achievement of Elementary Instrumental Music Students », thèse de doctorat, University of Rochester.

Azzara, Christopher D. (2002). «Improvisation», dans Richard Colwell (dir.), The New Handbook of Research on Music Teaching and Learning, New York, Schirmer Books, p. 171-187.

BAILEY, Derek (1993). Improvisation: Its Nature and Practice in Music, Cambridge (MA), Da Capo Press.

Bandura, Albert (1997). Self-Efficacy: The Exercise of Control, New York, Worth Publishers.

BARRETT, Frank J. (1998). «Coda - Creativity and Improvisation in Jazz and Organizations: Implications for Organizational Learning», Organization Science, vol. $9, \mathrm{n}^{\circ}$ 5, p. 605-622.

27 Traduction libre d' «associative generation ». 
Berkowitz, Aaron L. (2009). «Cognition in Improvisation: The Art and Science of Spontaneous Musical Performance », thèse de doctorat, Harvard University.

Berkowitz, Aaron L. et Daniel AnsARI (2008). «Generation of Novel Motor Sequences: The Neural Correlates of Musical Improvisation», NeuroImage, vol. 41, $\mathrm{n}^{\circ} 2$, p. 535-543.

Berkowitz, Aaron L. et Daniel Ansari (2010). «ExpertiseRelated Deactivation of the Right Temporoparietal Junction during Musical Improvisation», NeuroImage, vol. 49, no 1, p. 712-719.

BerLIner, Paul F. (1994). Thinking in Jazz: The Infinite Art of Improvisation, Chicago, University of Chicago Press.

Biasutti, Michele et Luigi Frezza (2009). «Dimensions of Music Improvisation», Creativity Research Journal, vol. 21, n 2-3, p. 232-242.

BROPHY, Timothy S. (2001). «Developing Improvisation in General Music Classes», Music Educators Journal, vol. 88, p. 34-53.

Carnovale, Norbert et Richard Dyer (s.d.). «Schuller, Gunther», dans Grove Music Online. Oxford Music Online, http://www.oxfordmusiconline.com/subscriber/ article/grove/music/A2258122, consulté le 26 août 2014.

Chamblee, Catherine Burnette (2008). « Cognitive Processes of Improvisation: Performers and Listeners in the Organ Tradition and Contemporary Gospel Styles », thèse de doctorat, University of Pennsylvania.

Clarke, Eric F. (1988). «Generative Principles in Music Performance», dans John A. Sloboda (dir.), Generative Processes in Music: The Psychology of Performance Improvisation and Composition, Oxford, Clarendon Press, p. 1-26.

Clarke, Eric F. (1992). «Improvisation, Cognition and Education», Companion to Contemporary Musical Thought, vol. 2, p. 787-802.

Covington, Kate. (1997). "Improvisation in the Aural Curriculum: An Imperative», College Music Symposium, vol. 37, 49-64.

Cropley, Arthur J. (2011). «Definitions of Creativity », dans Mark A. Runco et Steven R. Pritzker (dir.), Encyclopedia of Creativity (Second Edition), San Diego, Academic Press.

Csikszentminalyi, Mihaly et Grant Jewell Rich (1997). «Musical Improvisation: A Systems Approach», dans Richard Keith Sawyer (dir.), Creativity in Performance, Londres, Ablex, p. 43-66.

Dobbins, Bill (s.d.). «Peterson, Oscar », dans Grove Music Online. Oxford Music Online, http://www. oxfordmusiconline.com/subscriber/article/grove/ music/21443, consulté le 26 août 2014.
Dos Santos, Regina Antunes Teixeira et Luciana Del Ben (2004). "Contextualized Improvisation in Solfège Class», International Journal of Music Education, vol. 22, no 3 , p. 271-282.

Ericsson, K. Anders, Roy W. Roring et Kiruthiga Nandagopal (2007). "Giftedness and Evidence for Reproducibly Superior Performance: An Account Based on the Expert Performance Framework», High Ability Studies, vol. 18, n 1, p. 3-56.

Friberg, Anders et Andreas Sundström (2002). «Swing Ratios and Ensemble Timing in Jazz Performance: Evidence for a Common Rhythmic Pattern», Music Perception, vol. 19, n ${ }^{\circ} 3$, p. 333-349.

Gabrielsson, Alf (2003). «Music Performance Research at the Millennium», Psychology of Music, vol. 31, n 3, p. 221-272.

Gioia, Ted (1990). The Imperfect Art: Reflections on Jazz. and Modern Culture», New York, Oxford University Press.

GrIfFIths, Paul (s.d.). «Aleatory», dans Grove Music Online. Oxford Music Online, http://www.oxfordmusiconline. com/subscriber/article/grove/music/00509, consulté le 19 juin 2012.

Guilbault, Denise Marie (2009). «The Effects of Harmonic Accompaniment on the Tonal Improvisations of Students in First Through Sixth Grade», Journal of Research in Music Education, vol. 57, n 2, p. 81-91.

Hargreaves, David J., Conrad A. Cork et Tina Setton (1991). "Cognitive Strategies in Jazz Improvisation: An Exploratory Study », Canadian Journal of Research in Music Education, vol. 33, n 1 , p. 47-54.

Howe, Michael J. A., Jane W. Davidson et John A. SlobodA (1998). «Innate Talents : Reality or Myth?», Behavioral and Brain Sciences, vol. 21, n ${ }^{\circ}$ 3, p. 399-407.

Johnson-LAIRD, Philip N. (1991). «Jazz Improvisation: A Theory at the Computational Level», dans Peter Howell, Robert West et Ian Cross (dir.), Representing Musical Structure, New York, Academic Press, p. 291-325.

Johnson-Laird, Philip. N. (2002). «How Jazz Musicians Improvise», Music Perception, vol. 19, n 3, p. 415-442.

Kenny, Barry J. et Martin Gellrich (2002). «Improvisation », dans Richard Parncutt et Gary E. McPherson (dir.), The Science and Psychology of Music Performance, New York, Oxford University Press, p. 117-134.

Koutsoupidou, Theano et Hargreaves, David J. (2009). «An Experimental Study of the Effects of Improvisation on the Development of Children's Creative Thinking in Music », Psychology of Music, vol. 37, n 3, p. 251-278. 
Kozbelt, Aaron (2011). "Theories of Creativity», dans Mark A. Runco et Steven R. Pritzker (dir.), Encyclopedia of Creativity (Second Edition), San Diego, Academic Press, p. 473-479.

KRATUS, John (1991). «Growing with Improvisation», Music Educators Journal, vol. 78, n ${ }^{\circ}$, p. 36-40.

KRATUS, John (1995). «A Developmental Approach to Teaching Music Improvisation», International Journal of Music Education, vol. 1, p. 27-38.

L'ÉCUYER, René (1987). «L'analyse de contenu: Notion et étapes», dans Jean-Pierre Deslauriers (dir.), Les méthodes de la recherche qualitative, Québec, Les Presses de l'Université du Québec, p. 49-65.

Lehmann, Andreas C., John A. Sloboda et Robert H. Woody (2007). "Composition and Improvisation», dans Psychology for Musicians: Understanding and Acquiring the Skills, New York, Oxford University Press. p. 127-144.

Lemaire, Patrick et Ludovic Fabre (2005). «Strategic Aspects of Human Cognition: Implications for Understanding Human Reasoning», dans Maxwell J. Roberts et Elizabeth J. Newton (dir.), Methods of Though: Individual Differences in Reasoning Strategies, Hove et New York, Psychology Press, p. 11-56.

Limb, Charles J. et Allen R. Braun (2008). «Neural Substrates of Spontaneous Musical Performance: An FMRI Study of Jazz Improvisation », PLoS One, vol. 3, $\mathrm{n}^{\circ} 2$, p. 1-9.

Martinsen, Øyvind L., Geir Kaufmann et Adrian Furnham (2011). "Cognitive Style and Creativity», dans Mark A. Runco et Steven R. Pritzker (dir.), Encyclopedia of Creativity (Second Edition), San Diego, Academic Press, p. 214-221.

Mason, Colin M. (2005). «A Comparative and Historical Survey of Four Seminal Figures in the History of Jazz Education», thèse de doctorat, University of Texas.

McPherson, Gary E. (1993). «Evaluating Improvisational Ability of High School Instrumentalists», Bulletin of the Council for Research in Music Education, vol. 119, p. 11-20.

Mendonça, David et William A. Wallace (2004). «Cognition in Jazz Improvisation: An Exploratory Study», dans 26th Annual Meeting of the Cognitive Science Society, Chicago, Cognitive Science Society.

De Menezes, José Manuel Amaro (2010). «Creative Process in Free Improvisation », mémoire de maîtrise, University of Sheffield.

Monk, Augusto (2012). «The Five Improvisation "Brains": A Pedagogical Model for Jazz Improvisation at High School and the Undergraduate Level», International Journal of Music Education, vol. 30, n 2, p. 89-98.
Montano, David Ricardo (1983). "The Effect of Improvisation in Given Rhythms on Rhythmic Accuracy in Sight Reading Achievement by College Elementary Group Piano Students », thèse de doctorat, Conservatory of Music, University of Missouri.

Moore, Robin (1992). «The Decline of Improvisation in Western Art Music: An Interpretation of Change», International Review of the Aesthetics and Sociology of Music, vol. 23, n ${ }^{\circ}$ 1, p. 61-84.

Mumford, Michael D. et William B. Vessey (2011). «Heuristics: Strategies in Creative Problem Solving», dans Mark A. Runco et Steven R. Pritzker (dir.), Encyclopedia of Creativity (Second Edition), San Diego, Academic Press, p. 601-607.

NARDONE, Patricia L. (1996). «The Experience of Improvisation in Music: A Phenomenological, Psychological Analysis », thèse de doctorat, Saybrook Institute.

NetTl, Bruno (2009). "On Learning the Radif and Improvisation in Iran », dans Bruno Nettl et G. Solis (dir.), Musical Improvisation: Art, Education, and Society, Urbana, University of Illinois Press, p. 185-199.

Nettl, Bruno, Rob C. Wegman, Imogene Horsley, Michael Collins, Stewart A. Carter, Greer Garden, Robert E. Seletsky, Robert D. Levin, Will Crutchfield, John Rink, Paul Griffiths et Barry Kernfeld (s.d.). «Improvisation», dans Grove Music Online. Oxford Music Online, http://www.oxfordmusiconline.com/ subscriber/article/grove/music/13738, consulté le 10 décembre 2013.

NoRgaARD, Martin (2008). «Descriptions of Improvisational Thinking by Artist-Level Jazz Musicians », thèse de doctorat, University of Texas.

NorgaARD, Martin (2011). «Descriptions of Improvisational Thinking by Artist-Level Jazz Musicians », Journal of Research in Music Education, vol. 59, n 2, p. 109-127.

Plucker, Jonathan A., Mark A. Runco et C. Boyd Hegarty (2011). "Enhancement of Creativity», dans Mark A. Runco et Steven R. Pritzker (dir.), Encyclopedia of Creativity (Second Edition), San Diego, Academic Press, p. 456-460.

Pressing, Jeff (1984). «Cognitive Processes in Improvisation», dans Anthony J. Chapman et W. Ray Crozier (dir.), Cognitive Processes in the Perception of Art, vol. 19, North-Holland, Elsevier, p. 345-363.

Pressing, Jeff (1988). «Improvisation: Methods and Models», dans John A. Sloboda (dir.), Generative Processes in Music, Oxford, Oxford University Press, p. 129-178. 
Pressing, Jeff (1998). «Psychological Constraints on Improvisational Expertise and Communication», dans Bruno Nettl et Melinda Russell (dir.), In the Course of Performance: Studies in the World of Musical Improvisation, Chicago, University of Chicago Press, p. 47-67.

RowLYK, William Terry (2008). «Effects of Improvisation Instruction on Nonimprovisation Music Achievement of Seventh and Eighth Grade Instrumental Music Students », thèse de doctorat, Temple University.

Runco, Mark A. (2001). «Creativity and Cognition», dans Neil J. Smelser et Paul B. Baltes (dir.), International Encyclopedia of the Social et Behavioral Sciences, Oxford, Pergamon, p. 2892-2895.

Runco, Mark A. (2011). «Tactics and Strategies for Creativity», dans Mark A. Runco et Steven R. Pritzker (dir.), Encyclopedia of Creativity (Second Edition), San Diego, Academic Press, p. 423-426.

SARATH, Ed (1996). «A New Look at Improvisation», Journal of Music Theory, vol. 40, nº 1, p. 1-38.

SAWYER, Richard Keith (2000). "Improvisation and the Creative Process: Dewey, Collingwood, and the Aesthetics of Spontaneity», The Journal of Aesthetics and Art Criticism, vol. 58, $\mathrm{n}^{\circ}$ 2, p. 149-161.

SAWYER, Richard Keith (2011). «Improvisation», dans Mark A. Runco et Steven R. Pritzker (dir.), Encyclopedia of Creativity (Second Edition), San Diego, Academic Press, p. 647-652.

Scotт, Julie K. (2007). «Me? Teach Improvisation to Children?», General Music Today, vol. 20, n 2, p. 6-13.
SlaweK, Stephen. "Shankar, Ravi », dans Grove Music Online. Oxford Music Online, http://www. oxfordmusiconline.com/subscriber/article/grove/ music/25580, consulté le 26 août 2014.

Solis, Gabriel et Bruno NetTl (2009). Musical Improvisation: Art, Education, and Society, Urbana, University of Illinois Press.

Thompson, Sam et Andreas C. Lehmann (2004). «Strategies for Sight-Reading and Improvising Music », dans Aaron Williamon (dir.), Musical Excellence: Strategies and Techniques to Enhance Performance, Oxford, Oxford University Press, p. 143-159.

Vallerand, Robert J. et Ursula Hess (dir.) (2000). Méthodes de recherche en psychologie, Montréal, Gaëtan Morin.

WARD, Thomas B. (2007). «Creative Cognition as a Window on Creativity», Methods, vol. 42, n 1, p. 28-37.

Whitman, Georann Gale (2001). «The Effects of Vocal Improvisation on Attitudes, Aural Identification Skills, Knowledge of Music Theory, and Pitch Accuracy in Sight-Reading of High School Choral Singers», mémoire de maîtrise, Conservatory of Music, University of Missouri.

WiLson, Dorothy Stevens (1970). «A Study of the Child Voice from Six to Twelve », thèse de doctorat, University of Oregon.

Young, Susan (2003). «The Interpersonal Dimension: A Potential Source of Musical Creativity for Young Children? », Musicae Scientiae, vol. 7, numéro spécial, p. 175-191. 